Revista de Filosofía

Volumen 66, (2010) 5 - 23

\title{
LA FRASE DE HEIDEGGER “LA CIENCIA NO PIENSA”, EN EL CONTEXTO DE SU MEDITACIÓN SOBRE LA ERA TÉCNICA ${ }^{1}$
}

\author{
Jorge Acevedo Guerra \\ Universidad de Chile \\ joaceved@gmail.com
}

\begin{abstract}
Resumen
En una breve presentación inicial, hago notar la importancia de Heidegger y el reconocimiento que se le ha brindado, inclusive por parte de científicos de renombre. Luego, aludo a la tesis de Heidegger "la ciencia no piensa", a partir de ¿Qué significa pensar? A continuación, procuro aclarar tal aserto desde su conferencia "Ciencia y Meditación". Considero explicaciones posibles de la frase en cuestión, descartando varias de ellas y postulando que hay que pensar en otra dirección para dar cuenta de la tesis. Esa nueva dirección implica introducir la diferencia entre pensar calculador y meditación, así como centrarse en lo que Heidegger entiende por ser, esencia, sentido, esencia de la técnica moderna (Ge-stell: im-posición, conducto, dis-puesto, dispositivo) y Unicuadridad o Cuaternidad (Geviert).
\end{abstract}

Palabras Clave: Heidegger, ciencia, época técnica, pensar calculador, meditación, sentido, ser, esencia, Ge-stell, Unicuadridad.

\section{Abstract}

In a brief initial presentation, I underline the importance of Heidegger's thoughts and its acknowledgement by renowned thinkers and scientists. Then, I refer to Heidegger's thesis "science does not think" from the book What Calls for Thinking? or What is Called Thinking? In order to clarify this sentence, I use his lecture "Science and Reflection". Possible explanations of the phrase in question are put aside, and I postulate that is better to think in another direction in order to better understand the thesis. This new direction introduces the difference between calculative thinking and reflection (Besinnung, besinnliches Nachdenken: meditative thinking) and focuses on what Heidegger understands as: being, essence, sense, essence of modern technology (Ge-stell: enframing) and Fourfold (Geviert).

KEYWORDS: Heidegger, science, age of technology, calculative thinking, reflection, sense, being, essence, Ge-stell, Fourfold.

Este texto se inscribe dentro del Proyecto $\mathrm{N}^{\mathrm{o}} 1095168$ del Fondecyt de Chile, del cual el autor es el investigador responsable. 


\section{Heidegger, un clásico}

$\overline{R A}$ Uno de los filósofos más importantes de la historia del pensar de Occidente es Martin Heidegger. Lo afirma, por ejemplo, José Ortega y Gasset, quien no solía elogiar ligeramente, y menos aún a un coetáneo suyo. Poco antes de morir, casi con setenta años de edad, decía Ortega: "Heidegger es siempre profundo, y esto quiere decir que es uno de los más grandes filósofos que haya habido nunca"; y agregaba: "quiero parecerme a nuestro gran Heidegger, que no gusta, como los otros hombres, de detenerse sólo en las cosas, sino sobre todo -y esto es muy peculiar en él- en las palabras" (Ortega 2006a, pp. 802 y 811).

¿Por qué sería tan importante dentro de la historia de la filosofía? Sin pretender en lo más mínimo dar una respuesta cabal a esta pregunta, digamos por lo pronto, que recoge y reinterpreta la tradición occidental íntegra, desde Homero, Safo y Píndaro hasta Nietszche, Husserl, Scheler, Jaspers y Norbert Wiener, padre de la cibernética, pasando por las más grandes figuras de nuestra civilización: Newton, Galileo, Leibniz, Kant, Hegel. A esos nombres podemos agregar una larga lista de personajes que han contribuido a configurar el mundo en que vivimos, aunque, claro está, no nos demos cuenta de esa contribución.

La lista, sin embargo, probablemente no sea extremadamente larga. A pesar de su inmenso saber, Heidegger se concentró en algunos textos y en algunos asuntos decisivos. La erudición le era completamente ajena. Solo abordó lo más radical, lo más profundo, lo que nos determina desde el fondo del acontecer histórico y de la existencia personal. Después de su encuentro con Heidegger en el Château de Cerisy, en agosto de 1955, Julián Marías comentaba: "Heidegger, hombre quizás de no demasiadas lecturas, iqué hondo y tenaz lector de sus griegos, de sus idealistas alemanes, de su Hölderlin!” (Marías 1961, p. 417; lo cursivo es mío).

Después de Heidegger, todo se transfigura. Tenemos otro Platón, otro Aristóteles, otro Nietzsche, otra noción de filosofía, otra idea de la verdad, otra perspectiva sobre el hombre, otra concepción de la ética, otra interpretación de la ciencia moderna, otra versión de la religión, otro modo de habérselas con el pensar, otra vinculación con el lenguaje, otra aproximación al ser, otra visión de Dios.

No considerar suficientemente su aporte al pensamiento contemporáneo podría implicar, creo, el riesgo de haberse quedado retrasado, permanecer en lo que filosóficamente es insuficiente, en concepciones de las cosas que han quedado atrás dentro del ámbito del pensar esencial. Por otra parte, me parece -solo es una impresión mía-, que pretender haberlo superado es dar muestra de una incomprensión de su grandeza, o de ingenuidad, o de una autoestima excesiva. Como la de todo gran filósofo, su obra espera seguir siendo trabajada, de tal modo de extraer de ella "la integridad" de lo que puede dar. La extracción de su riqueza no es asunto de un par de meses ni de un par de años ; tampoco en algunas décadas se logrará que nos hagamos cargo de su legado. La ocupación con su herencia va para largo y, sin duda, quedará como un clásico de la filosofía. Es decir, como uno de aquellos autores a los que necesariamente hay que volver una y otra vez, porque el nivel de problematismo en que han logrado moverse 
nos exige un diálogo constante con ellos, so pena de perder contacto con lo que más nos importa. Heidegger es un clásico en el sentido que da Ortega a esta palabra. "Lo clásico -dice-, no es lo ejemplar ni lo definitivo: no hay individuo ni obra humana que la humanidad, en marea viva, no haya superado. (...) Ni nuestra caritativa admiración ni una perfección ilusoria y "eterna" hacen al clásico, sino precisamente su aptitud para combatir con nosotros. (...) Ahora bien: esto no sería posible si el clásico no hubiese calado hasta el estrato profundo donde palpitan los problemas radicales. Porque vio algunos claramente y tomó ante ellos posición, pervivirá mientras aquellos no mueran. (...). El error está en creer que los clásicos lo son por sus soluciones. Entonces no tendrían derecho a subsistir, porque toda solución queda superada. En cambio el problema es perenne. Por eso no naufraga el clásico" cuando el pensamiento progresa (Ortega 2006b, pp. 230 s.).

Como es sabido, su primer y más conocido aporte es habernos proporcionado una nueva interpretación del ser humano, lo que efectúa en su libro Ser y tiempo, en el cual concibe al ser humano como Dasein, como existir, como ser-ahí (en la traducción al español de la palabra Dasein hecha por José Gaos -gran discípulo de Ortega-, después de haber dedicado veinte años a verter al castellano esa obra). El Dasein consiste ante todo en estar-en-el-mundo y no solo en pensar -tomando esta palabra en un sentido estrecho-, como había afirmado Descartes con su célebre apotegma cogito, ergo sum, "pienso, luego existo". Fijar la mirada en el estar-en-el-mundo nos hace caer en la cuenta de que nuestra vida cotidiana tiene una especial relevancia, aunque normalmente la pasemos por alto y no nos fijemos en ella. Nuestro estar-en-elmundo se da, inmediata y regularmente, en el modo de la cotidianidad. Desde ella se erigen, sin perder nunca contacto con ella, modalidades especiales de la existencia; la artística: del pintor, del escultor, del arquitecto o del poeta ; la teorética: del científico o del filósofo; la política: del hombre de Estado; la religiosa: del devoto, del sacerdote, del teólogo.

Ser y tiempo y otros escritos del autor ha tenido ya un gran impacto en prácticamente todas las esferas del saber (Xolocotzi 2008). Cuando Heidegger cumple ochenta años de edad, el filósofo alemán Richard Wisser recoge testimonios de reconocimiento hacia su obra de grandes representantes de las más diversas disciplinas. De un físico, Carl-Friedrich von Weizsäcker -iniciador del Instituto Max Planck para la investigación de las condiciones de vida del mundo científico-técnico, de Munich-, quien fuera ayudante de Werner Heisenberg. De filósofos europeos, Maurice de Gandillac, Leo Gabriel, Karl Löwith. De un filósofo y escritor, Ernst Jünger. De un filósofo japonés, Kôichi Tsujimura. De un estudioso de la literatura, el filólogo Emil Staiger. De un médico psiquiatra, Medard Boss, continuador -en la línea de Heidegger-, de Ludwig Binswanger y de Freud. De un teólogo protestante, Heinrich Ott, y de uno católico, Karl Rahner.

Su meditación de nuestra época como la era del predominio de la técnica moderna parece de la mayor importancia. Tengo la impresión, nuevamente, de que desconocer esta meditación impediría darse cuenta cabalmente de lo que ocurre tanto en nuestro mundo histórico como en nuestra vida personal, ya que tanto el uno como la otra están decisivamente condicionados por la técnica moderna tal como la entiende 
Heidegger. La llamada globalización -de que tanto se habla en nuestros días-, no sería sino una manifestación derivada del predominio de la técnica moderna. Derivada, digo, puesto que tal predominio sería un fenómeno meta-económico (Fédier 1995, p. 209). Por cierto, no es fácil decir en pocas palabras en qué consiste y qué alcances tiene la meditación heideggeriana de la técnica. He dedicado algún tiempo a reflexionar sobre este "diagnóstico" de la época actual que hace Heidegger, y parte de los resultados de esa reflexión la he recogido en mi libro Heidegger y la época técnica, publicado por la Editorial Universitaria de Santiago. También he editado un libro de Heidegger, que con el título Filosofía, Ciencia y Técnica, recoge textos de la mayor relevancia sobre el asunto: "La pregunta por la técnica", "La Vuelta", "Construir Habitar Pensar”, "Ciencia y Meditación”, "Tiempo y Ser”, "La cosa”, “¿A qué se llama pensar?" Su quinta edición aparece con el sello de la Editorial Universitaria el año 2007.

Sin postular de manera ilusa que el pensamiento de Heidegger o la filosofía son la panacea universal, creo que muchos problemas del hombre y de las sociedades de hoy pueden abordarse fructíferamente desde su perspectiva, con la esperanza de que así puedan recibir algo así como una "solución", en la medida en que se pueda hablar de "soluciones" cuando se trata de las cuestiones más graves de la existencia y de su abordaje a través del pensar, dentro de una época de crisis como la nuestra.

\section{La ciencia no piensa}

¿Por qué Heidegger dice que la ciencia no piensa? La pregunta no es fácil de contestar. Sobre todo, si se quiere ser entendido al responderla.

Un lugar clásico en que Heidegger señala que la ciencia no piensa es la lección primera de su curso ¿A qué se llama pensar? o ¿Qué significa pensar? "Esta afirmación -indica allí mismo-, resulta escandalosa". Pero no se arredra ante el escándalo, y prosigue así: "dejemos a la frase su carácter escandaloso, aun cuando apostillemos inmediatamente que, no obstante, la ciencia tiene que habérselas con el pensar en su propia forma especial" (Heidegger 2005a, p. 19 /H. 2002, p. 9).

Sin duda, la tesis de Heidegger resulta chocante. Sin embargo, él no la enuncia de manera ingenua. Tampoco ignora las objeciones que, de inmediato, podrían hacerse a su postura. Comencemos con una obvia objeción, a la que alude en el tránsito de la primera a la segunda lección de ¿Qué significa pensar? "En la Universidad -indica-, es particularmente grande el peligro de entender mal lo que se refiere al pensamiento, en especial cuando se habla en forma explícita de las ciencias. Pues ¿en qué otro lugar se nos pide que nos rompamos la cabeza tanto como en los centros de investigación y docencia relacionados con el trabajo científico" (Heidegger 2005a, p. 76 /H. 2002, pp. 35 s.). Heidegger sería incoherente e injusto con la ciencia. Reconoce que en el trabajo científico las personas se rompen la cabeza como en ninguna otra parte. Estaría aceptando tácitamente, pues, que allí hay pensamiento, y de manera eminente. No 
obstante, insiste en que la ciencia no piensa; al parecer, con la más completa falta de ecuanimidad frente a la ciencia.

Pero, ¿hay, en efecto, falta de coherencia y de justicia con la ciencia? El mismo Heidegger dice que no. Refiriéndose a su propia meditación respecto de la ciencia hace esta aclaración: "Un pensador piensa solamente cuando persigue lo que habla $a$ favor de una cosa. Todo hablar a la defensiva sólo tiene el sentido de proteger la cosa. En la medida en que en nuestro camino hemos de hablar de las ciencias, no nos pronunciamos contra ellas, sino a favor de ellas, a saber, en pro de la claridad acerca de su esencia. Y en ello está contenida ya la persuasión de que las ciencias son en sí algo esencialmente positivo. No obstante, su esencia no es tal como tiende a representarla la mentalidad de nuestras universidades" (Heidegger 2005a, p. 76 /H. 2002, p. 16).

Cuando Heidegger afirma que la ciencia no piensa no se está pronunciando contra ella, sino a su favor, esto es, habla en pro de la claridad acerca de la esencia de la ciencia. Hablar en pro de la claridad de la esencia de la ciencia supone que ésta es algo esencialmente positivo. "Cuando se establece una diferencia, una distinción y separación entre el pensar y la ciencia -nos advierte-, eso se considera de inmediato como una degradación de la ciencia. E incluso se teme que el pensamiento inicie las hostilidades contra la ciencia y perturbe la seriedad y el agrado en el trabajo científico" (Heidegger 2005a, p. 76 /H. 2002, p. 16).

Tal consideración y tal temor nacen de un malentendido y originan un malentendido. Sería necesario superarlos.

Pero Heidegger añade algo más. Es preciso recordar que el libro en que hallamos la frase de que la ciencia no piensa recoge el último curso que dio en la Universidad de Friburgo antes de ser nombrado profesor emérito. Por tanto, su auditorio estaba lleno de científicos. Dada tal circunstancia, indica que los temores ante una posible actitud hostil del pensamiento contra la ciencia no son fundados, agregando que "en el caso de que lo fueran, carecería de tacto y gusto quien se alzara contra la ciencia en el recinto destinado a formarse en la ciencia. Por razones de tacto habría de evitarse aquí toda polémica. Y hemos de añadir otro motivo. En todo tipo de polémica se pierde de antemano la actitud del pensamiento. La función de un contradictor no es la del pensamiento" (Heidegger, 2005a: 76/H., 2002: 16). Heidegger, pues, no es ni un desatinado carente de tacto ni un contradictor de la ciencia; es un pensador respetuoso.

Pero, ¿es una afirmación pensante y respetuosa decir que la ciencia no piensa? ¿No parece todo lo contrario? Esa apariencia engañosa habría que disiparla. Por lo pronto, indicando en qué sentido restringido toma Heidegger la palabra pensar en su afirmación sobre la ciencia.

La palabra pensar en este contexto se refiere al pensar filosófico. "Los filósofos -dice Heidegger-, son los "pensadores". Así se llaman porque propiamente el pensamiento tiene su escenario en la filosofía" (Heidegger 2005a, p. 16 /H. 2002, p. 6). Por tanto, podemos llegar a una primera y modesta conclusión que esclarece un tanto la chocante determinación de la ciencia antes indicada. La ciencia no piensa en el sentido del pensar filosófico. 
En una entrevista efectuada en 1969, Richard Wisser le hace una pregunta a Heidegger que le permite a éste precisar el alcance de su famosa frase sobre la ciencia. La pregunta es formulada así: "Dos cosas son siempre puestas por usted en cuestión y puestas como cuestionables: la pretensión de dominio de la ciencia y una comprensión de la técnica que no ve en ella sino un medio apto para llegar más rápidamente a una meta deseada. Justamente en nuestro tiempo, en que los hombres esperan todo de la ciencia y en el que se les demuestra en transmisiones de televisión mundiales que el hombre alcanza por la técnica lo que se proponga, justamente en este tiempo, sus pensamientos sobre las ciencias y sobre la esencia de la técnica causan muchos dolores de cabeza; en primer lugar, ¿qué quiere decir usted cuando afirma: la «ciencia no piensa»?"

La respuesta de Heidegger: "Para comenzar primero con los dolores de cabeza. Los encuentro muy sanos. Hoy día hay demasiado pocos dolores de cabeza en el mundo y una gran falta de pensamiento que justamente va con el olvido del ser. Y esa frase, la ciencia no piensa, que causó gran sensación cuando la dije en una lección en Friburgo, significa: la ciencia no se mueve en la dimensión de la filosofía, pero está, sin que ella lo sepa, referida a esa dimensión. Por ejemplo, la física se mueve en espacio, tiempo y movimiento; lo que sea movimiento, lo que sea espacio, lo que sea tiempo, no lo puede decidir la ciencia en cuanto [als] ciencia; entonces, la ciencia no piensa; no puede en este sentido pensar con sus métodos. Yo no puedo, por ejemplo, con métodos físicos decir lo que sea la física. Lo que sea la física yo sólo lo puedo pensar a la manera del preguntar filosófico. La frase la ciencia no piensa no es ninguna objeción, sino solamente una constatación de la estructura interna de la ciencia; a su esencia pertenece que ella esté referida, por una parte, a lo que piensa la filosofía y, por otra parte, ella misma, sin embargo, olvida esto que hay que pensar y no lo considera" (Heidegger 1980, p. 176 /H. 1971, pp. 75 s. /H. 1970, pp. 71 s. Lo cursivo de en cuanto es mío).

La ciencia no piensa ni puede pensar con sus propios métodos lo que está en la base de lo que se ha llamado sus conceptos fundamentales, que corresponden al ámbito al que está referida (Precisiones, en: Heidegger 2007a, pp. 181 ss. /H. 1994a, pp. 56 ss. /H. 2000a, pp. 60 ss.). Esto no quiere decir que los científicos no piensen y ni siquiera puedan pensar en tales asuntos. Pueden hacerlo, y, de hecho, lo hacen y, al parecer, muy bien además. Pero esta tarea no la llevan a cabo poniendo en juego los métodos de las ciencias, sino planteando preguntas en un estilo filosófico. En esto reside una vinculación decisiva de la ciencia con la filosofía, la que habitualmente es desatendida.

Por cierto, en este planteo no se está proponiendo que la filosofía sea superior a las ciencias; menos aún se sugeriría una superioridad personal del filósofo sobre el científico.

Que el físico no pueda definir la física mediante una medición propia del método de la física no significa que no pueda hacerlo en absoluto. Le "basta" con abandonar el método científico y adoptar una actitud filosófica para procurar llegar a una determinación de su ciencia. 
Probablemente, esta tarea no es fácil de realizar, dado que, como indica Heidegger, habría que reconocer "el abismo que media entre el pensar [filosófico] y las ciencias", abismo "que media entre ambos polos como infranqueable. Aquí no hay ningún puente -agrega-, hay solamente un salto" (Heidegger 2005a, p. 19 /H. 2002, p. 10).

El habérselas de la ciencia con el pensar en su propia forma especial solo sería auténtica y, en consecuencia, fructífera, a través de ese salto (Heidegger 2005a, p. 19 /H. 2002, p. 10). Por eso -añade Heidegger-, son perjudiciales todos los puentes provisionales y los puentes de vía estrecha que precisamente hoy quieren instalar un cómodo tráfico recíproco entre el pensar y las ciencias" (Heidegger 2005a, p. 19/H. 2002, p. 10).

Pero no querría desviarme del tema principal. ¿En qué sentido la ciencia no piensa?

\section{Los límites de la ciencia: "Ciencia y Meditación"}

En un breve ensayo, "Ciencia y Meditación", Heidegger nos indica que una manifestación de que la ciencia no piensa reside en que no está en condiciones de autodefinirse recurriendo a los métodos que pone en juego en cuanto ciencia. Da ejemplos que pueden resultar esclarecedores. "La Física - dice-, en cuanto Física no puede hacer ninguna afirmación sobre la Física. Todas las afirmaciones de la Física hablan físicamente. La Física misma no es un objeto de posibles experimentos físicos. Lo mismo vale para la Filología. En cuanto teoría de la lengua y de la literatura no es nunca un objeto posible que pueda ser tratado filológicamente. Lo dicho vale para toda ciencia" (Heidegger 2007a, p. 180 /H. 1994a, p. 46 /H. 2000a, p. 59).

El mismo Heidegger se encarga de ponerse un problema. Dice: "Pero podría hacerse una objeción: la Historiografía en cuanto ciencia, al igual que las restantes ciencias, tiene una historia. Entonces, la ciencia histórica puede tratarse [u observarse] a sí misma, en el sentido de su temática y de su método" (Heidegger 2007a, pp. $180 \mathrm{~s}$. /H. 1994a, p. 47 /H. 2000a, pp. 59 s.). Heidegger reconoce que es así. Pero indica que ese autotratamiento o autoobservación tiene un límite decisivo: no alcanza lo esencial. $\mathrm{Y}$ da otro ejemplo - muy esclarecedor, en mi opinión-, para reafirmar su tesis. Afirma: "Ciertamente. La Historiografía abarca [o aprehende] por medio de tal tratamiento [u observación] la ciencia que ella es. Sólo que la Historiografía no abarca [o no aprehende] nunca con eso su esencia en cuanto historiografía, es decir, en cuanto ciencia. Si se quiere afirmar algo sobre la matemática en cuanto teoría -este sería el ejemplo especialmente esclarecedor-, entonces se tiene que abandonar el campo de objetos de la Matemática y su modo de concebir. No se puede nunca establecer por medio de un cálculo matemático qué sea la Matemática en sí misma" (Heidegger 2007a, p. 181/H. 1994a, p. 47 /H. 2000a, p. 60).

Así como las ciencias no pueden autodefinirse mediante sus métodos -eso es lo que hemos querido establecer o sugerir-, tampoco pueden a través de ellos definir 
suficientemente aquello sobre lo que versan. La naturaleza, en el caso de la física; la historia, en el caso de la historiografía; el hombre, en el de la psiquiatría; la lengua, en el de la filología. Todo eso -naturaleza, historia, hombre, lengua-, pertenece a lo que para las ciencias queda como inabarcable o irrodeable. "Si las ciencias -dice Heidegger-, por sí mismas pudieran encontrar en sí lo inabarcable citado, tendrían ante todo que estar en condiciones de concebir su propia esencia. Pero -como lo establecimos o sugerimos antes-, ellas no están nunca en condición de hacerlo" (Heidegger 2007a, p. 180 /H. 1994a, p. 46 /H. 2000a, p. 59).

Tomemos el ejemplo de la psiquiatría. Dice Heidegger: "La Psiquiatría trata de la vida psíquica humana en sus manifestaciones patológicas y, por tanto, sanas. Ella concibe esto desde la objetidad [u obstancia: Gegenständigkeit] de la unidad corporal-espiritual-psíquica de todo el hombre. En la objetidad [u obstancia] de la Psiquiatría se ex-pone en cada caso la existencia [Dasein] humana ya presente. El ser-ahí [Da-sein] en donde ec-siste [ek-sistiert] el hombre en cuanto hombre, queda como lo inabarcable para la Psiquiatría" (Heidegger 2007a, p. 178 /H. 1994a, p. 45 /H. 2000a, pp. $57 \mathrm{~s}$.). ¿Qué quiere decir esto? Ciertamente, no se está diciendo que la psiquiatría carece de toda noción sobre el hombre Sin duda, tiene vastos conocimientos acerca de él. Probablemente, más que la filosofía. Sin embargo, el vínculo entre el hombre y lo que Heidegger llama el ser queda al margen de esa disciplina como tal. Como ese vínculo -la ec-sistencia (Ek-sistenz)-, es decisivo para Heidegger, puede afirmar que a la psiquiatría en cuanto tal se le escapa lo más propio del hombre y que, por tanto, el hombre queda para ella como lo irrodeable o inabarcable (Unumgängliche).

Hago resaltar este ejemplo porque dentro de "Ciencia y Meditacion" es breve. Además, porque Heidegger trabajó durante varios años -desde 1959 hasta 1969-, con un grupo de psiquiatras encabezados por Medard Boss en una nueva fundamentación filosófica de la psiquiatría. Al comienzo tropezó con la incomprensión, antipatía y rechazo de una serie de médicos afincados en la "ciencia pura" y el psicoanálisis. No obstante, con el paso del tiempo, esas resistencias fueron atenuándose. Hoy funciona, con sede en Suiza, una escuela psiquiátrica que se basa en lo que se ha llamado Análisis del Dasein o Análisis de la existencia (Daseinsanalyse), el cual se funda en la analítica existencial o existenciaria del Dasein (existenziale Analytik des Daseins) expuesta en Ser y Tiempo y, en general, en el pensamiento de Heidegger. Los escritos reunidos bajo el título Seminarios de Zollikon, traducidos por Ángel Xolocotzi, son el documento más importante respecto de este punto. Medard Boss atestigua en ellos que Heidegger vio en sus encuentros con los psiquiatras con los que trabajó durante más de una década "la posibilidad de que sus intuiciones filosóficas no permanecieran inmóviles en las aulas de los filósofos, sino que pudieran beneficiar a numerosos seres humanos y sobre todo también a los necesitados". Al mismo tiempo, añade Boss, "con su actitud frente a nuestro círculo de Zollikon demostró que no solamente sabía decir y escribir de la forma suprema de la proximidad, sino que él también estaba dispuesto a vivirla de manera ejemplar. Él ejemplificaba el cuidado desinteresado y amoroso que se anticipa al otro ser humano dejándolo en libertad" (Heidegger 2007b, pp. 14 s. /H. 1994b, XII s.). 
4. Hipótesis desde las cuales se trata de explicar que la ciencia no piensa. Pensar calculador y pensar meditativo

Retomo lo anterior. Si lo irrodeable o inabarcable queda para la ciencia como lo inaccesible, el ser mismo -el asunto del pensar filosófico-, también permanece inaccesible a la ciencia como tal. Lo irrodeable inaccesible (o lo inabarcable inaccesible: das unzugängliche Unumgängliche) constituye para Heidegger lo que denomina "situación latente" o "estado de cosas imperceptible" o "estado de cosas inadvertido" (unscheinbare Sachverhalt) de la ciencia (Heidegger 2007a, pp. 179 ss. /H. 1994a, pp. 57 ss. /H. 2000a, pp. 58 ss.). Heidegger se limita a indicar acerca de ello en "Ciencia y Meditación". Utilizando un par de metáforas señala hacia esa situación o estado de cosas situado en el paraje donde enraizaría la esencia de la ciencia. Dice: "La situación latente -o estado de cosas inadvertido-, se oculta en las ciencias. Pero no está en ellas como la manzana en el cesto. Más bien tendríamos que decir: las ciencias, por su parte, descansan en la situación latente -o estado de cosas inadvertido [unscheinbare Sachverhalt]-, como el río en su fuente" (Heidegger 2007a, p. 183 /H. 1994a, p. 58 /H. 2000a, p. 62).

Añade Heidegger: "Nos contentamos con indicar hacia la situación latente. Para determinar lo que sea ésta en sí misma, se exigiría un nuevo preguntar. Sin embargo, ahora y mediante la indicación hacia la situación latente, estamos referidos a un camino que conduce hacia lo digno de ser preguntado [das Fragwürdige]" (Heidegger 2007a, pp. 183 s. /H. 1994a, pp. 58 s. /H. 2000a, pp. 62 s.).

Para Heidegger, lo digno de ser preguntado por antonomasia es el ser. También el ser queda inaccesible para la ciencia como tal.

Podríamos plantear algunas hipótesis para tratar de explicar lo dicho anteriormente.

Primera hipótesis: la situación latente o estado de cosas inadvertido podría ser visto, aunque sin ser propiamente observado, "porque se considera insuficientemente y rara vez la esencia de la ciencia" (Heidegger 2007a, pp. 181 s. /H. 1994a, p. 57 /H. 2000a, p. 60). Heidegger considera débil la última parte de la hipótesis. "Esto último -a saber, que no se considera con suficiente frecuencia la esencia de la ciencia-, apenas podría afirmarlo nadie con fundamento - advierte. Al contrario: muchos testimonios hablan a favor de que hoy en día, no sólo por la Física sino por todas las ciencias pasa una extraña inquietud". Más aún: "antes, en los pasados siglos de la historia occidental del espíritu y de las ciencias, se hicieron reiterados ensayos para delimitar la esencia de las ciencias. El vehemente e incesante esfuerzo en esto es, sobre todo, un rasgo fundamental de la Edad Moderna. Entonces, ¿cómo pudo quedar inobservada [o inadvertida] aquella situación [o estado de cosas]?” (Heidegger 2007a, p. 182 /H. 1994a, p. 57 /H. 2000a, pp. 60 s.).

Volviendo su vista hacia la Época Contemporánea, insiste Heidegger "en las múltiples dilucidaciones que se han hecho [actualmente] sobre las ciencias. Hoy en día -añade-, se filosofa sobre las ciencias desde los más diversos puntos de vista. En estos esfuerzos, por parte de la Filosofía nos encontramos con las autopresentaciones 
que están intentando las ciencias por todas partes, en forma de esbozos sintéticos y contando la historia de la ciencia" (Heidegger 2007a, p. 182 /H. 1994a, pp. 57 s. /H. 2000a, p. 61). En esta misma línea de pensamiento, Ortega advierte en su curso ¿Qué es filosofia?, de 1929, que ha sido paradójico "que después de tanta teoría del conocimiento fabricada por los filósofos tuvieran que encargarse los físicos mismos de dar la última precisión al carácter de su conocimiento" (Ortega 2008, p. 259).

Por ende, queda descartada la hipótesis según la cual la situación latente queda inadvertida para la ciencia debido al poco interés por la esencia de la ciencia y a los escasos intentos que se habrían efectuado para dilucidar tal esencia por parte de la filosofía y de la misma ciencia.

Una segunda hipótesis: la situación latente -en que está inmersa la ciencia como el río en su fuente-, y lo más digno de ser preguntado -el ser mismo-, no nos llaman la atención, de tal modo que los hombres no nos fijamos ni en la una ni en el otro (Heidegger 2007a, pp. 182 s. /H. 1994a, p. 58 /H. 2000a, pp. 61 s.). Esta hipótesis responsabilizaría al ser humano de la ocultación de esas instancias. Cierta negligencia suya sería la causa de la falta de saber respecto de la situación latente y del ser en general. Heidegger considera equivocada esta hipótesis. Respecto de la situación latente, nos dice que su latencia no se funda en una conducta cognoscitiva defectuosa del hombre. "Lo latente de la situación latente-afirma-, se funda más bien en que ésta no aparece por sí misma. En lo inabarcable inaccesible en cuanto tal yace el que se la pase continuamente por alto" (Heidegger 2007a, p. 183 /H. 1994a, p. 58 /H. 2000a, p. 61. Lo cursivo es mío).

Algo semejante ocurre respecto del ser mismo, y no solo en su relación con la ciencia; "el hecho de que no pensemos -señala Heidegger en su libro ¿Qué significa pensar?-, de ninguna manera se debe a que el hombre [-no sólo la ciencia-,] todavía no se dirija en medida suficiente a lo que originalmente quisiera ser pensado, que en su esencia permanece como lo que ha de pensarse. Nuestra tardanza procede, más bien, de que lo merecedor mismo de ser pensado [-el ser-,] se aparta del hombre" (Heidegger 2005a, p. 18 /H. 2002, p. 8).

Por tanto, en el seno del asunto planteado hay un desplazamiento: la ocultación del estado de cosas inadvertido y del ser mismo no se debe solo ni principalmente a factores puramente humanos -descuido, negligencia, esfuerzos mal orientados, empresas teoréticas malogradas-, sino, más bien, a que el uno y el otro tienden a la ocultación; más todavía; para Heidegger, lo más propio del ser mismo consiste en su quedar retraído, en su apartarse del hombre. En ese sentido interpreta el fragmento 123 de Heráclito: Physis kryptesthai philê̂: "Lo más propio del brotar en ser es su quedar retraído" (Soler, "Prólogo". En: Heidegger 2007a, p. 57 - Fédier, 2008, pp. 82 s.).

Una tercera hipótesis: la ciencia no alcanza los estratos más profundos de lo real -esto es, el ser mismo-, porque su esfuerzo pensante ha sido mal dirigido y deficientemente realizado. Heidegger se opone frontalmente a ella. La ciencia no alcanza el ser mismo porque no puede alcanzarlo dada su propia estructura interna, la que la inscribe en lo que Heidegger llama pensar calculante (rechnendes Denken). Por 
definición, al pensar calculante o técnico le está negado el acceso al ser como tal. Para aclarar este punto recurriremos a la conferencia de Heidegger titulada Serenidad.

Pero antes hagamos un breve puente hacia ella a través de la conferencia que hemos venido citando. En "Ciencia y Meditación" se alude a la serenidad (Gelassenheit). "Meditación -leemos allí-, es la serenidad respecto de lo digno de ser preguntado" (Heidegger 1994a, p. 59 /H. 2007a, p. 184 /H. 2000, p. 63). Y explicitando eso, dice Heidegger: "Seguir el camino que un asunto ya ha tomado por sí mismo, se dice en alemán sinnan, sinnen. Introducirse en el sentido [Sinn] es la esencia de la meditación [Besinnung]" (Heidegger 2007a, p. 184 /H. 1994a, p. 59 /H. 2000a, p. 63).

En Serenidad entra en mayores detalles a propósito de la confrontación entre pensar calculante y pensar meditativo. Refiriéndose al primer tipo de pensar -el calculante-, dice: "Su peculiaridad consiste en que cuando planificamos, investigamos, organizamos una empresa, contamos ya siempre con circunstancias dadas. Las tomamos en cuenta con la calculada intención de unas finalidades determinadas. Contamos de antemano con determinados resultados. Este cálculo caracteriza a todo pensar planificador e investigador. Semejante pensar sigue siendo cálculo aun cuando no opere con números ni ponga en movimiento máquinas de sumar ni calculadoras electrónicas. El pensamiento que cuenta, calcula; calcula posibilidades continuamente nuevas, con perspectivas más ricas y a la vez más económicas. El pensamiento calculador no es un pensar meditativo" (Heidegger 1994c, p. 18 /H. 2000c, pp. 519 s.).

El pensar meditativo (das besinnliche Nachdenken), frente al calculador, se caracteriza por pensar "en pos del sentido [Sinn] que impera en todo cuanto es" (Heidegger 1994c, p. 18 /H. 2000c, p. 520).

La ciencia sería un modo del pensar calculador. No se trata, pues, de que en ella haya una ausencia absoluta de pensamiento. Lo que no hay en ella, en tanto ciencia, es un pensar meditativo.

"Hay así dos tipos de pensar -agrega Heidegger-, cada uno de los cuales es, a su vez y a su manera, justificado y necesario: el pensar calculador y el pensar meditativo [das rechnende Denken und das besinnliche Nachdenken]" (Heidegger 1994c, p. $18 /$ H. 2000c, p. 520).

Se ve, pues, que Heidegger no adopta una actitud desdeñosa frente al pensar calculador; es justificado y necesario; sin él no podríamos vivir y, por tanto, el pensar meditativo sería imposible. No se trata, por tanto, de contraponerlos de una manera excluyente, sino de distinguirlos de un modo integrativo dentro de nuestra existencia.

Por ende, la frase "la ciencia no piensa" quedaría así, en principio, explicada y suficientemente matizada; habríamos mostrado, brevemente y en bosquejo, su alcance y sus límites.

Tendríamos que añadir, destacándolo, que el hecho de que la ciencia no piense, en el sentido ya indicado, es una ventaja decisiva para ella, no un inconveniente. "El hecho de que la ciencia no pueda pensar [o no sepa pensar] -dice Heidegger-, no es una carencia [o un defecto], sino una ventaja. Esta ventaja le asegura a la ciencia la 
posibilidad de introducirse en cada zona de objetos según el modo de investigación y de instalarse en aquélla" (Heidegger 1994a, p. 117 /H. 2007a, pp. 270 s. /H. 2000a, p. 133).

Insiste Heidegger: "la ciencia [...] no piensa ni puede pensar, y, por cierto, para su propio bien, o sea, para asegurar la propia marcha que ella se ha fijado" (Heidegger 2005a, p. 19 /H. 2002, p. 9). Y luego establece de manera sorprendente, a primera vista, una jerarquización inesperada y una diferenciación rotunda entre ciencia y pensar. En sus palabras: "La esencia de los respectivos ámbitos [de las ciencias] -la historia, el arte, la poesía, el lenguaje, la naturaleza, el hombre, Dios-, permanece inaccesible a las ciencias. Y a la vez éstas caerían constantemente en el vacío si no se movieran dentro de sus propios ámbitos. La esencia de tales ámbitos es el asunto del pensamiento. En cuanto las ciencias como tales [als Wissenschaften] no tienen ningún acceso a ese asunto, hemos de decir que ellas no piensan. Lo dicho suena en un primer momento a cierta presunción, como si el pensamiento se tuviera a sí mismo por superior a las ciencias. Esa presunción sería injustificada allí donde se diere. Pues, precisamente porque el pensamiento se mueve allí donde podría pensar la esencia de la historia, del arte, del lenguaje, de la naturaleza, pero se mueve allí en tal manera que todavía no es capaz de pensarla, en consecuencia el pensamiento sabe mucho menos que las ciencias. Éstas exhiben su nombre con pleno derecho, pues saben infinitamente más que el pensar" (Heidegger 2005a, p. 76 /H. 2002, pp. 35 s.).

\section{Esencia, ser, Unicuadridad, Ge-stell}

Hay algunas cuestiones de especial importancia sobre las cuales vuelvo. En primer lugar, sobre la esencia. ¿Qué es lo esencial? Ciertas personas -algunos filósofos de las ciencias y algunos científicos, entre ellas-, suelen molestarse ante esta palabra. Sospechan que cuando se habla de esencia no se está hablando de nada o, al menos, de nada inteligible. ¿De qué se está hablando cuando buscamos la esencia? Heidegger habla mucho de la esencia. Recuérdese, por ejemplo, su Parménides (Heidegger $2005 \mathrm{~b} / \mathrm{H}$. 1982). Por lo mismo, en varios lugares procura aclarar lo que quiere decir con esa palabra. En sus conferencias sobre "La esencia del habla" se enfrenta a este asunto. "Este título - dice-, «La esencia del habla», suena más bien presuntuoso, como si aquí fuera cuestión de dar a conocer información certera sobre la esencia del habla. Además, el título se presenta formalmente de modo casi demasiado familiar, como: la esencia del arte, la esencia de la libertad, la esencia de la técnica, la esencia de la verdad, la esencia de la religión, etc. Estamos ya cansados de la acumulación de esencias, por motivos que no acabamos de percibir del todo" (Heidegger 1990, p. 156 /H. 1985, p. 164). El mismo Heidegger se siente inquieto, pues, del problema implícito en el uso de la palabra esencia, el cual, por cierto, no pretendemos resolver ahora. Sin embargo, queremos recoger algunas señales que da el filósofo al respecto.

En esas mismas conferencias, Heidegger recuerda que "el «qué es» (Wassein), contiene desde Platón lo que habitualmente denominamos das Wesen, essentia, la esencia de una cosa. La esencia así entendida se enmarca en lo que más tarde se 
denomina der Begriff, el concepto, die Vorstellung, la representación, con la ayuda de los que nos procuramos y asimos lo que una cosa es". Pero Heidegger quiere desprenderse de esas nociones de esencia, y propone entender esencia de otra manera, a saber, "como verbo; wesend, «esenciante», en el sentido de presente y ausente (Wesend wie anwesend und abwesend). «Esencia» significa perdurar, permanecer. Con todo, la expresión es west, lo «esenciante», dice más que sólo: esto perdura y permanece. Es west quiere decir: esto «esencia» en presencia (es west an) y perdurando nos concierne, nos en-camina y nos de-manda. La esencia entendida de este modo nombra lo que perdura (das Währende), lo que viene hacia nosotros y en todo nos concierne porque en-camina" (Heidegger 1990, p. 180 /H. 1985, pp. 189 s.).

Esta manera de asumir la esencia podría desconcertarnos. Más bien, es desconcertante; a menos que la insertemos en la totalidad del pensamiento de Heidegger. Es lo que muy brevemente hace François Fédier al traducir el título de estas conferencias como "El despliegue de la palabra" (Heidegger 1976a, pp. 141, 11, 203). Pero, ¿a qué nos referimos con despliegue? ¿Qué es lo que en última instancia se despliega? ¿Qué es lo que piensa el pensamiento cuando se dirige hacia lo esencial? Hay algo que, por así decirlo, ostenta de manera eminente los cuatro caracteres de la esencia, de lo que se despliega. Estos caracteres son: 1. Perdura. 2. Viene hacia nosotros. 3. Nos concierne en todo. 4. En-camina. Lo que ostenta estos caracteres es lo que Heidegger llama el ser. El ser es lo que en primera y última instancia constituye la esencia. Más aún: sugiere Heidegger que, quizás (vielleicht), constituya la única esencia (Heidegger 2007a, p. 149 /H. 1994a, p. 33 /H. 2000, p. 32). Por tanto, todas las esencias remitirían finalmente a lo que tal vez sea lo único que dura (währt) encaminándonos: el ser, la esencia.

Legítimamente, puede llamar la atención el primer carácter nombrado: la duración. ¿Es que Heidegger vuelve al viejo substancialismo, después de haberlo rechazado con energía en Ser y tiempo? Fédier nos da una explicación iluminadora al dilucidar más ampliamente lo que quiere decir Heidegger con la palabra Wesen. "Cuando da indicaciones sobre [el substantivo] «Wesen»-advierte-, Heidegger lo aproxima de buen grado al verbo «währen» [durar] que no es, de hecho, sino el durativo del [verbo] «wesen». Basta con considerar esto: durar, en sentido pleno, no implica en modo alguno la inmutabilidad (¿es necesario agregar: todo lo contrario?) -basta con haber notado esto para comenzar a ver el abismo que separa la esencia (en sentido tradicional) de lo que Heidegger entiende con «Wesen»-" (Fédier 2008, pp. 77 s.).

En las conferencias antes aludidas, Heidegger destaca una destinación del ser: la Unicuadridad (das Geviert). Habría que considerar otra, a la que alude sin nombrarla: la im-posición o con-ducto (das Ge-stell). Abordemos esto.

La Unicuadridad, Cuaternidad, cuadrante o cuaterna nombra la reunión de los cuatro: cielo y tierra, mortales y divinos; o tierra y cielo, dios y hombre (Heidegger 1990, p. 192 /H. 1985, p. 202). Se trata probablemente de la destinación primordial y matriz del ser. Heidegger habla de las regiones de la cuaternidad del mundo, de las cuatro regiones del mundo o de la Cuaternidad del mundo (Heidegger 1990, pp. 189 s., 193 /H. 1985, p. 203). El juego del mundo es lo que perdura, viene hacia nosotros, 
nos concierne en todo, nos en-camina. Pensar lo esencial es pensar el ser, es dejarse interpelar por el juego del mundo, correspondiéndole. No se trata, pues, de captar intuitivamente algo, ni menos aún de capturar conceptualmente la Unicuadridad. Se trata, en otras palabras, de "retornar adonde (propiamente) ya nos hallamos" (Heidegger 1990, p. $170 /$ H. 1985, p. 180). Por cierto, la ciencia como tal nada tiene que ver con el pensar así entendido.

Para referirse al ser como la Unicuadridad, Heidegger escribe la palabra ser tachándola con un aspa o cruz de San Andrés. Con esa grafía quiere decir que no se refiere al ser tradicionalmente entendido; dicho en breve, no apunta en absoluto al ser como objeto. Pero hay más. "Esa tachadura en forma de aspa-explica-, en principio sólo trata de impedir la costumbre -casi imposible de erradicar- de representarnos al ser como algo que está y subsiste por sí mismo y de cuando en cuando aparece frente al hombre. De acuerdo con esta representación, parece como si el hombre estuviese excluido fuera del «ser». Pero no sólo no está fuera, es decir, no sólo está comprendido dentro del «ser», sino que, usando al ser humano, el «ser» se ve abocado a renunciar a la apariencia del para-sí, motivo por el que tiene otra esencia distinta de la que le gustaría a la representación de un tipo de compendio que abarca la relación sujeto-objeto. Después de lo dicho, el signo del aspa no puede ser un signo meramente negativo de tachadura. Por el contrario, lo que hace es señalar las cuatro regiones del conjunto integrado por los cuatro y su reagrupamiento en el punto en que se cruza el aspa" (Heidegger 2000b, pp. 332 s. /H. 1976b, p. 411). A estas cuatro regiones se refiere más ampliamente en "Construir Habitar Pensar", "La cosa” y “. . . poéticamente habita el hombre..." (Heidegger 1994a /H. 2000a).

Recojamos este planteo: el hombre como mortal, como el habitante, no está fuera del ser y frente a él-como el sujeto frente al objeto-, sino que se inscribe en el ser, habita en él. Precisamente, esta unión de ser y hombre (o Dasein) es el acontecimiento apropiador o de transpropiación (Ereignis). Y así como se puede ver esto a propósito del ser como la Unicuadridad, también es posible verlo a propósito del ser como das Gestell, la im-posición, el con-ducto. En "El principio de identidad", Heidegger lo muestra. Allí indica, hablando de la im-posición, la esencia de la técnica moderna, que "el juego de propiación en que el hombre y el ser se transpropian recíprocamente" es lo que nombra Ereignis (Heidegger 1988a, pp. 84 ss.). En "El camino al habla" aborda también el asunto: "En la medida en que el con-ducto (Ge-stell) desafía al hombre, o sea, lo reta a atender (bestellen) a todo lo presente como un inventario (Bestand) técnico, la unidad de todos los modos de puesta en posición-el con-ducto-, se despliega (west) según el modo del acontecimiento apropiador o de transpropiación (Ereignis) y esto de tal manera que al mismo tiempo disimula (verstellt) a este último porque todo cometido (Bestellen) se ve remitido al pensamiento calculador y así habla el lenguaje del con-ducto. El habla está desafiada a corresponder en todos los sentidos a la disponibilidad (Bestellbarkeit) técnica de todo lo presente" (Heidegger 1990, p. $238 /$ H. 1985, pp. 251 s. Traducción ligeramente modificada). Espero que lo que acabo de decir se clarifique con lo que agregaré más adelante sobre lo Gestell. En cualquier caso, traigo a colación lo que relata Jean Beaufret acerca de Ereignis; hacia el final del último seminario de Le Thor, en 1969, Heidegger le hizo la siguiente declaración 
incidental: Desde la Carta sobre el humanismo, continúo diciendo Sein, ser, pero pienso Ereignis, acontecimiento apropiador (Beaufret 1985, p. 126).

Tal como dejamos establecido, las cuatro regiones del ser están configuradas por la tierra, el cielo, los divinos y los mortales. Pensar lo cuadrante, la cuaterna, la cuaternidad o la Unicuadridad es el habitar al que Heidegger aspira y al que nos invita. "En el salvar a la Tierra, en el acoger al Cielo, en el esperar a los Divinos, en el guiar de los Mortales, se acontece [ereignet sich: acaece de un modo propio] el habitar en cuanto cuádruple proteger [Schonen: cuidar, mirar por] de lo cuadrante. Proteger [cuidar, mirar por] quiere decir: custodiar lo cuadrante en su esencia. Pero - pregunta Heidegger-, ¿dónde guarece [o guarda] el habitar, cuando protege lo cuadrante, la esencia de éste? (...) El habitar como proteger [cuidar, mirar por] guarece [o guarda] lo cuadrante en donde los mortales se mantienen: en las cosas" (Heidegger 2007a, p. 215 /H. 1994a, p. 133 /H. 2000, p. 153). Pensar el ser, por uno de sus lados -la palabra ser va en este caso tachada por un aspa-, y genuino habitar son lo mismo (aunque no lo igual).

La invitación a pensar el ser como la Unicuadridad o habitar genuinamente en su seno no deja fuera a nadie. Las personas que no adhieren a una religión o que no creen en Dios también pueden asumir ese habitar de que habla Heidegger. En principio, con las mismas facilidades y dificultades de alguien que pertenece a una iglesia o es un devoto creyente. Sin duda, precisar en detalle cómo sería el habitar en un caso y en el otro sería muy problemático, suponiendo que tales precisiones puedan hacerse. De todas maneras, a lo divino que hay en el ser y en el hombre -siguiendo una sugerencia de Hölderlin recogida por Fédier-, se le puede llamar con el nombre al que cada cual pueda recurrir, y se le puede asumir de la manera que corresponda a tal nombre (Fédier 2007, pp. 16 s.).

Pensar lo esencial no se agota en lo indicado. Implica también -y con esto tampoco se agota-, pensar la im-posición, das Ge-stell, esto es, como ya señalamos, la esencia de la técnica moderna. Esta palabra ha sido traducida de varias maneras para recoger lo que quiere decir. Ge-stell: im-posición (Manuel Olasagasti, Adolfo P. Carpio, Héctor Delfor Mandrioni), dis-puesto (Francisco Soler, Francisco Ugarte), posición-total (Jorge Eduardo Rivera), dispositivo (Jorge Mario Mejía), in-stalación (Germán Bleiberg), estructura-de-emplazamiento (Eustaquio Barjau), disposición (Marcos García de la Huerta), com-posición (Helena Cortés y Arturo Leyte), lo compuesto-en-disposición-de uso (Rafael Ángel Herra), con-ducto (Ángel Xolocotzi). André Préau vierte al francés por arraisonnement [apresamiento, inspección], que significaría "reconducir de grado o por fuerza a la razón" (Fédier 2008, p. 79), lo que, por cierto, alude a la concepción heideggeriana de la ratio. Das Ge-stell es un destino del ser, Geschick des Seins (Heidegger 2005c, p. 276 /H. 1977, p. 371 -Heidegger, 1991, pp. 105 ss. /H. 1997, pp. 89 ss.). Dicho brevemente, con Fédier: "Destino -si este fenómeno es bien visto-, no es otra cosa que el hecho de dirigirse a alguien (potencialmente a todos, en la medida en que son concernibles), exigiendo de él una respuesta" (Fédier 2008, 36).

Gestell "es un término que no puede ser traducido en forma literal -indica Xolocotzi. Tal como se señala en «La pregunta por la técnica», añade, Gestell indica 
la congregación de modos de presencia en la metafísica occidental y no sólo de la época técnica. (...) Mi propuesta es conducto precisamente porque así como el stellen en el término «Gestell» congrega los modos de presencia, así ducere ha señalado un camino en las lenguas romances que corresponde al fenómeno señalado: el conducto sería la congregación de los modos de presencia dados en Occidente y que conocemos como producción, inducción, reducción, etc." (En: Heidegger 2007b, pp. 241 s., en nota). En este caso sucedería lo mismo que ocurre con la Unicuadridad, a saber: 1. No es posible intuir la esencia; el ser no se da en una intuición. ¿Alguien podría intuir ese complejo fenómeno histórico que, de algún modo, recoge la historia de Occidente? 2. Pensar el conducto no puede consistir en apoderarse de él a través del concepto (Begriff), lo que nos daría un dominio sobre él y la posibilidad de manejarlo a nuestro gusto o según nuestras conveniencias. Aquí no hay un agarrar (greifen), sino algo completamente diferente. 3. La ciencia no piensa ni puede pensar de esa manera distinta, no conceptual. ¿En qué podría consistir el pensar de que hablamos?

Algo ya hemos dicho sobre eso: 1. En la interpelación del ser en que nos encontramos sumidos es preciso "retornar adonde (propiamente) ya nos hallamos". 2. Hay que asumir como conviene la correspondencia en que ya estamos respecto de la interpelación del ser. ¿Dónde nos hallamos? Tal como propone François Fédier, nos encontramos en el reino soberano de la verdad, la que se manifiesta como desocultar pro-vocante (herausforderndes Entbergen) -inherente al habitar técnico que se da bajo el predominio de la im-posición-, y como un desocultar respetuoso, protector, custodiante, cuidadoso, albergador -inherente a lo que podríamos llamar un genuino habitar. La tarea del pensar consistiría, por lo pronto -como indica Fédier interpretando a Heidegger-, en ductibilizarse, hacerse flexible y "apto para tomar su forma a partir del reino soberano de la verdad y del esfuerzo que requiere el hecho de tomarla en custodia" (Fédier 2008, p. 89). Asumir como conviene la correspondencia en que ya estamos dentro de la interpelación del ser como Gestell no consiste en obedecer pasiva y ciegamente la conminación de la im-posición o con-ducto, sino en adquirir esa ductibilización y flexibilización que nos permita ver la técnica como "verdad que nos atañe, es decir, que nos requiere -sin la menor conminación-que, en reciprocidad, la tomemos en custodia. Pero tomar en custodia, en la época en que estamos -advierte Fédier-, no es posible de otro modo sino recomenzando cada día todo el itinerario de nuevo. Tal es la proeza de perseverancia que requiere de nosotros hoy día la comprensión de la técnica" (Fédier 2008, p. 90).

\section{Meditación (Besinnung) y sentido (Sinn)}

El segundo punto que quiero tocar muy brevemente está emparentado con el anterior. Es el sentido. El pensar meditativo -que no es el científico-, va tras el sentido del acontecer. La determinación de que lo es sentido que hay en Ser y tiempo implica estar familiarizado con la terminología de todo ese libro. Por eso adujimos otra, de "Ciencia y Meditación". Recordemos el texto: "Seguir el camino que un asunto ya ha tomado por sí mismo, se dice en alemán sinnan, sinnen. Introducirse 
en el sentido [Sinn] es la esencia de la meditación [Besinnung]" (Heidegger 2007a, p. 184 /H. 1994a, p. 59 /H. 2000a, p. 63). Pero hay otra determinación en la que se dice aproximadamente lo mismo acerca de la palabra sinnan, y que nos ayuda a entender mejor el asunto. "«Sinnan»-señala Heidegger, explicando la palabra sentido (Sinn)-, significa originariamente: viajar, aspirar a..., tomar una dirección. La raíz indogermánica sent y set significa el camino" (Heidegger 1990, p. 50 /H. 1985, p. 49). Meditación sería, pues, pensar en la dirección del camino en el que el ser mismo nos ha encaminado, introduciéndose como conviene en ella -la dirección-, y en él -el camino-- La meditación -pensar el sentido-, recae, pues, sobre el ser, sobre la esencia. No es algo diferente al pensar mismo, al pensar del ser, al pensar esencial, al corresponder como conviene a la interpelación del ser. De diversas maneras, Heidegger va confluyendo siempre sobre lo Mismo. Pero este desembocar en lo Mismo -que podría parecer simple monotonía-, confirma una de las grandes diferencias entre ciencia y pensamiento, a la que se refiere Heidegger en estos términos: "El investigador necesita siempre nuevos descubrimientos y ocurrencias, pues de otro modo la ciencia es presa del moho y de lo falso. El pensador necesita tan sólo un único pensamiento" (Heidegger, 2005a: 39 s. /H., 2002: 53). Por eso puede agregar, caracterizando su pensamiento: "desde lo Mismo y hacia lo Mismo, lo Mismo" (Heidegger 2007a, p. 319 /H. 1988b, p. 24. Citado en: Soler 1983, p. 82, n. 8).

\section{Referencias bibliográficas}

Beaufret, J. (1985), Dialogue avec Heidegger. IV. Le chemin de Heidegger, Paris: Les Éditions de Minuit.

Fédier, François (2008), Entendre Heidegger et autres exercices d'écoute, Paris: Le Grand Souffle. (2007), Voix de l'ami. Paris: Éditions du Grand Est.

(1995), Regarder Voir. Paris: Les Belles Lettres / Archimbaud.

Heidegger, M. (2007a), Filosofía, Ciencia y Técnica. Edición y prólogo de Jorge Acevedo. Trad. y prólogo de Francisco Soler. Santiago de Chile: Universitaria.

(2007b), Seminarios de Zollikon. Protocolos-Diálogos-Cartas. Edición de Medard Boss, Trad. de Ángel Xolocotzi Yáñez. Morelia, Michoacán, México: Red Utopía Asociación Civil / Jitanjáfora Morelia (vierte una edición más completa -del año 2006-, que la señalada más adelante). Trotta.

(2005a), ¿Qué significa pensar? Trad. de Raúl Gabás Pallás. Madrid:

(2005b), Parménides. Trad. de Carlos Másmela. Madrid: Akal.

(2005c), Caminos de bosque. Trad. de Helena Cortés y Arturo Leyte. Madrid: Alianza.

(2002), Gesamtausgabe, 8: Was heißt denken? Edición de PaolaLudovika Coriando. Frankfurt a. M.: Klostermann. 
(2000a), Gesamtausgabe, 7: Vorträge und Aufsätze. Edición de FriedrichWilhelm von Herrmann. Frankfurt a. M.: Klostermann.

(2000b), Hitos. Trad. de Helena Cortés y Arturo Leyte. Madrid: Alianza.

(2000c), Gesamtausgabe,16: Reden und andere Zeugnisse eines Lebensweges. Edición de Hermann Heidegger. Frankfurt a. M.: Klostermann.

(1997), Gesamtausgabe, 10: Der Satz vom Grund. Edición de Petra Jaeger. Frankfurt a. M.: Klostermann.

(1994a), Conferencias y Artículos. Trad. de Eustaquio Barjau. Barcelona: del Serbal.

(1994b), Zollikoner Seminare: Protokolle - Zwiegspräche - Briefe. Edición de Medard Boss. Frankfurt a. M.: Klostermann.

(1994c), Serenidad. Trad. de Yves Zimmermann. Barcelona: del Serbal.

(1991), La proposición del fundamento. Trad. de Félix Duque y Jorge Pérez de Tudela. Barcelona: del Serbal.

(1990), De camino al habla. Trad. de Yves Zimmermann. Barcelona: del Serbal.

(1988a), Identidad y diferencia / Identität und Differenz. Trad. de Helena Cortés y Arturo Leyte. Ed. bilingüe de Arturo Leyte. Barcelona: Anthropos.

(1988b), Zur Sache des Denkens. Tübingen: Max Niemeyer.

(1985), Gesamtausgabe, 12: Unterwegs zur Sprache. Edición de FriedrichWilhelm von Herrmann. Frankfurt a. M.: Klostermann.

(1982), Gesamtausgabe, 54: Parmenides. Edición de Manfred S. Frings. Frankfurt a. M.: Klostermann.

(1980), "Martin Heidegger en diálogo", en: García de la Huerta I., M., La Técnica y el Estado Moderno. Santiago: Departamento de Estudios Humanísticos de la Universidad de Chile.

(1977), Gesamtausgabe, 5: Holzwege. Edición de Friedrich-Wilhelm von Herrmann. Frankfurt a. M.: Klostermann.

(1976a), Acheminement vers la parole. Trad. de Jean Beaufret, Wolfgang Brokmeier y François Fédier. París: Gallimard.

(1976b), Gesamtausgabe, 9: Wegmarken. Edición de Friedrich-Wilhelm von Herrmann. Frankfurt a. M.: Klostermann.

(1971), "Martin Heidegger dialoga”, en: Wisser, R. (Ed.), Martin Heidegger al habla. Trad. de Don Eloy Requena y Rafael Ángel Herra. Madrid: Stvdivm.

(1970), “Martin Heidegger im Gespräch”, en: Wisser, R. (Ed.), Martin Heidegger im Gespräch. Freiburg/München: Karl Alber.

Marías, J. (1961), Obras. Tomo VI. Madrid: Revista de Occidente.

Ortega y Gasset, J. (2008), Obras Completas. Vol. VIII. Madrid: Taurus /Fund. J.O.G. 
(2006a), Obras Completas. Vol. VI. Madrid: Taurus /Fund. J.O.G.

(2006b), Obras Completas. Vol. V. Madrid: Taurus /Fund. J.O.G.

Soler, F. (1983), Apuntes acerca del pensar de Heidegger. Edición de Jorge Acevedo Guerra. Santiago de Chile: Andrés Bello.

Xolocotzi, Á. (2008), “Martin Heidegger”, en: Erik Ávalos (coordinador), Pensador@s del siglo XX, México, IMCED-Trillas. Disponible en:

http://angelxolocotzi.com/page14.php (recuperado el 20 de septiembre de 2009).

Advertencia: En las referencias a textos de Heidegger $(\mathrm{H})$, indico primero la versión en castellano, y luego la versión en alemán, separándolas con un /. Si recurro a más de una versión en castellano, señalo primero aquélla que he utilizado íntegramente - o como base- en la cita correspondiente. Los volúmenes de la Edición integral citados son versiones revisadas, con notas marginales que el autor escribió en su ejemplar de la obra. 